OPEN ACCESS

Edited by:

Lars Neeb,

Charité - Universitätsmedizin

Berlin, Germany

Reviewed by:

Michele Viana,

King's College London,

United Kingdom

Mamoru Shibata,

Tokyo Dental College Ichikawa

General Hospital, Japan

Allison M. McKendrick,

The University of Melbourne, Australia

*Correspondence:

Christoph J. Schankin christoph.schankin@insel.ch

Specialty section:

This article was submitted to Headache and Neurogenic Pain,

a section of the journal

Frontiers in Neurology

Received: 11 June 2021

Accepted: 31 August 2021

Published: 04 October 2021

Citation:

Klein A and Schankin CJ (2021) Visual Snow Syndrome as a Network Disorder: A Systematic Review.

Front. Neurol. 12:724072.

doi: 10.3389/fneur.2021.724072

\section{Visual Snow Syndrome as a Network Disorder: A Systematic Review}

\author{
Antonia Klein and Christoph J. Schankin* \\ Department of Neurology, Inselspital, Bern University Hospital, University of Bern, Bern, Switzerland
}

\begin{abstract}
Aim: By reviewing the existing clinical studies about visual snow (VS) as a symptom or as part of visual snow syndrome (VSS), we aim at improving our understanding of VSS being a network disorder.

Background: Patients with VSS suffer from a continuous visual disturbance resembling the view of a badly tuned analog television (i.e., VS) and other visual, as well as non-visual symptoms. These symptoms can persist over years and often strongly impact the quality of life. The exact prevalence is still unknown, but up to $2.2 \%$ of the population could be affected. Presently, there is no established treatment, and the underlying pathophysiology is unknown. In recent years, there have been several approaches to identify the brain areas involved and their interplay to explain the complex presentation.
\end{abstract}

Methods: We collected the clinical and paraclinical evidence from the currently published original studies on VS and its syndrome by searching PubMed and Google Scholar for the term visual snow. We included original studies in English or German and excluded all reviews, case reports that did not add new information to the topic of this review, and articles that were not retrievable in PubMed or Google Scholar. We grouped the studies according to the methods that were used.

Results: Fifty-three studies were found for this review. In VSS, the clinical spectrum includes additional visual disturbances such as excessive floaters, palinopsia, nyctalopia, photophobia, and entoptic phenomena. There is also an association with other perceptual and affective disorders as well as cognitive symptoms. The studies that have been included in this review demonstrate structural, functional, and metabolic alterations in the primary and/or secondary visual areas of the brain. Beyond that, results indicate a disruption in the pre-cortical visual pathways and large-scale networks including the default mode network and the salience network.

Discussion: The combination of the clinical picture and widespread functional and structural alterations in visual and extra-visual areas indicates that the VSS is a network disorder. The involvement of pre-cortical visual structures and attentional networks might result in an impairment of "filtering" and prioritizing stimuli as top-down process with subsequent excessive activation of the visual cortices when exposed to irrelevant external and internal stimuli. Limitations of the existing literature are that not all authors used the ICHD-3 definition of the VSS. Some were referring to the symptom VS, and in many cases, the control groups were not matched for migraine or migraine aura.

Keywords: visual snow syndrome, systematic (literature) review, perceptual disorder, electrophysiology, imaging, network disorder, neuroophtalmology, visual disturbance 


\section{INTRODUCTION}

The first description of the visual snow phenomenon (VS) was presented by Liu et al. in 1995 with four patients with migraine who had interictal visual disturbances (1). Initially, these symptoms were thought to be persistent migraine aura $(2,3)$. Through the collection of 22 patient reports, Schankin et al. (4) noticed that the symptoms are very characteristic and not as polymorphous as they can be in typical migraine aura $(5,6)$. This was confirmed in larger groups via an internet survey (235 persons) and an additional semi-structured telephone interview (142 persons with self-assessed VS) (4, 7). It became evident that this disorder also affects patients who have not been diagnosed with migraine $(4,8)$. Finally, the group concluded that the symptom VS is often associated with additional visual, non-visual, and non-perceptual symptoms forming the visual snow syndrome (VSS), which is distinct from migraine. Based on this, diagnostic criteria have been proposed (4) that have been implemented in the international classification of headache disorders, 3rd edition (ICHD-3) $(4,9)$. The aim of this review is to summarize what we have learned about the underlying pathophysiology of VSS from clinical presentation, secondary forms, neurobehavioral studies and imaging, as well as electrophysiological testing.

\section{METHODS}

We performed a systematic review on PubMed (accessed May 1, 2021) and Google Scholar (accessed May 15, 2021) using the search term "visual snow."

We included case reports and studies describing epidemiological, neurophysiological, and imaging findings about the VSS or VS. We excluded articles not written in English or German, non-original work, such as reviews, case reports not adding information to the question of this review, and papers that could not be retrieved via Google Scholar or PubMed.

The records were screened by AK and also evaluated by CJS in respect of the inclusion and exclusion criteria.

Papers released prior to the publication of the diagnostic criteria for the VSS by the International Headache Society in 2018 (9) were not excluded when the criteria could be assessed retrospectively based on the clinical information presented.

Additionally, we performed a non-systematic search for the terms "palinopsia," "floaters," "blue field phenomenon," "selflight of the eye," "photopsia," "Nyctalopia," "Photophobia," "Lamotrigine" AND "migraine aura," "thalamostriatal loop," "thalamocortical dysrhythmia," and "salience network" to provide definitions of these expressions used in our review.

\section{RESULTS}

In total, we found 801 papers. After excluding doubles $(n=$ $107)$ and articles that were not on the topic $(n=543)$ of this review, there were 151 remaining of which 125 could be retrieved. Finally, we included 53 articles (Figure 1). We organized the articles according to the main topic and the used methodology.

\section{The Clinical Picture}

Several studies have collected data on the prevalence of symptoms in groups of patients with VSS or VS, either in online surveys $(4,11,12)$ or in interviews at headache centers or neuroophthalmologic departments (4,13-17). Per definition, patients with VS experience a visual static, similar to the flickering of an old TV, with many, moving colored or black-andwhite dots in the entire visual field. To fulfill the diagnostic criteria of the ICHD-3, there must be at least two additional characteristic symptoms:

1. Palinopsia is an abnormal, continued perception of an object even after it is no longer in the visual field $(18,19)$. This symptom is reported in 33\% (14) to $86 \%$ (4) of patients with VSS. it can manifest as a steady afterimage or as "trailing," i.e., positive afterimages persisting directly after a dynamic object (20).

2. Another group of symptoms are enhanced entoptic phenomena, which are thought to be perceptions of endogenic structures/phenomena of the eye (21):

- Floaters can be caused by degenerative changes in the vitreous body including liquefaction $(22,23)$. According to the above-mentioned studies, between $61 \%$ (16) and 100\% (24) of VSS patients are perceiving them in an excessive way, i.e., far more than normal vitreous floaters and far more than expected from ophthalmological findings.

- The so-called "blue field phenomenon" is characterized by a perception of moving fragments against a bright background, such as the sky (25). It is the autovisualization of the own leukocytes in the retinal capillaries (26). It has been shown that about $42 \%$ (27) to $79 \%$ (4) of VSS patients experience this symptom, also in an excessive magnitude.

- The "selflight of the eye" is light or colored clouds seen with closed eyes (26) and perceived by $16 \%$ (16) to $71 \%$ (11) of VSS patients. The cause of this phenomenon is unknown. Bowen et al. hypothesized that it might be linked to retinal circulation (26).

- Photopsia, reported in $24 \%(15,16)$ to $63 \%(4,11)$ of VSS patients, are "flash-like" positive visual phenomena with sudden onset and brief duration (28). The origin in different diseases can be generated by almost all components of the visual path from ocular mechanical disturbances to affections of the secondary visual pathways (28).

3. Nyctalopia: Patients complain about poor vision in darker environments. In the literature, it has often been described in patients with retinal diseases (especially affecting the rod cells), but the inability to adapt to light conditions might involve almost all components of the visual pathway (29). About $28 \%$ (16) to $78 \%$ (11) of VSS patients are affected.

4. Photophobia, which is experienced by $44 \%$ (16) to $81 \%$ (11), is hypersensitivity, discomfort, or even pain caused by "normal" light $(27,30)$. Eren et al. (27) compared a group of 19 VSS patients to a group of 19 controls matched for age, sex, migraine, and aura using the Leiden Visual Sensitivity Scale (31). They demonstrated that VSS patients had increased 


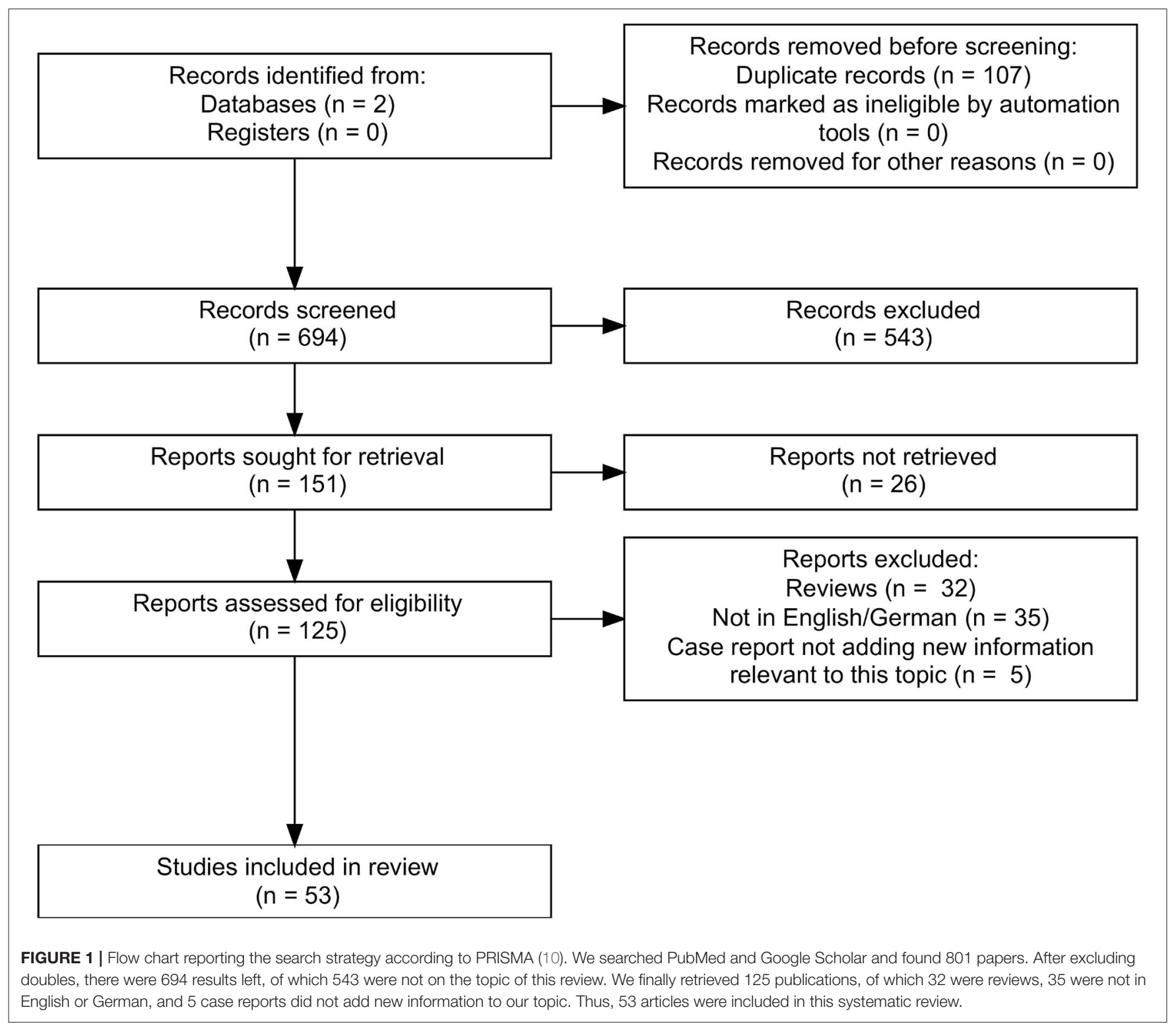

visual sensitivity at a level comparable to patients with chronic migraine during their attacks (27).

\section{Prevalence and Course of Disease}

Graber et al. conducted the first longitudinal study in VSS. Symptoms were persistent, in some cases up to 8 years (32). In several studies, there was a fraction of patients reporting VSS for as long as they could remember $(4,11,14)$. The data so far suggest that the VSS does often become a chronic disease. There are also published cases of episodic VS as part of a migraine attacks, but different from aura (33).

The first study to assess the prevalence of VSS within the population was by Kondziella et al. who used an online survey with 1,015 participants. Although this study has its limitations (34), a larger proportion of the general population, $2.2 \%$ in this study, might fulfill the criteria of VSS (35).

\section{Associated Symptoms and Disorders}

Between 52\% (13) and 72\% (11) of patients with VSS also suffer from migraine. In several clinical studies, patients reported migraine attacks in association with the appearance or the aggravation of their VSS $(4,11,14,16,36)$. Schankin et al. (4) and Puledda et al. (11) explored the relationship between migraine status and the manifestation of VSS and found that people with migraine tended to have more symptoms as a marker of a stronger affection by this disease. Another highly prevalent comorbidity affecting between $15 \%$ (14) and 75\% (11) of patients is tinnitus. Puledda et al. showed that for the VSS patients in their cohort who were also affected by tinnitus, the probability of having additional visual symptoms was 2 fold increased (11). Mehta et al. reported that $7.1 \%$ of their cohort with VS had a diagnosis of fibromyalgia (16). Additionally, postural orthostatic tachycardia syndrome (16), dizziness (16), balance problems 
TABLE 1 | Summary of the imaging findings.

\begin{tabular}{|c|c|c|c|}
\hline Modality & Study & $\begin{array}{l}\text { Patients/ } \\
\text { controls }\end{array}$ & Main findings \\
\hline FDG-PET & $\begin{array}{l}\text { Schankin } \\
\text { et al. (34) }\end{array}$ & $20 / 20$ & $\begin{array}{l}\text { - Hypermetabolism in the right lingual gyrus } \\
\text { - Hypometabolism in the right superior temporal gyrus and the left inferior parietal lobule }\end{array}$ \\
\hline \multirow[t]{3}{*}{$\begin{array}{l}\text { MRI } \\
\text {-Volumetry }\end{array}$} & $\begin{array}{l}\text { Schankin } \\
\text { et al. (34) }\end{array}$ & $17 / 17$ & $\begin{array}{l}\text { Global analysis: } \\
\text { - GMV increased: junction of the right lingual and fusiform gyrus, right middle temporal gyrus, right parahippocampal } \\
\text { gyrus, left superior temporal gyrus, right anterior cingulate cortex } \\
\text { - GMV decreased: left superior temporal gyrus }\end{array}$ \\
\hline & $\begin{array}{l}\text { Puledda et al. } \\
(11)\end{array}$ & $24 / 24$ & $\begin{array}{l}\text { Whole brain morphology, parcellated cerebellar analyses, } R O \text {-analyses: } \\
\text { - GMV increased: left V1(WB), left V1/V2 area (ROI), left V5 area (ROI), crus I/lobule VI of the left cerebellar hemisphere }\end{array}$ \\
\hline & $\begin{array}{l}\text { Aldusary et al. } \\
\text { (69) }\end{array}$ & $19 / 16$ & $\begin{array}{l}\text { Voxel-based morphometry: } \\
\text { - GMV increased: right lingual gyrus, visually: occipital bending in } 7 \text { patients }\end{array}$ \\
\hline \multirow[t]{3}{*}{ fMRI } & $\begin{array}{l}\text { Puledda et al. } \\
(11)\end{array}$ & $24 / 24$ & $\begin{array}{l}\text { MRI block-design (visual stimulation) with MRS: } \\
\text { - Reduced BOLD responses: left and right anterior insula, MRS lactate-Peak over right gyrus lingualis with } \\
\text { anticorrelation to BOLD response }\end{array}$ \\
\hline & $\begin{array}{l}\text { Puledda et al. } \\
\text { (68) }\end{array}$ & $24 / 24$ & $\begin{array}{l}\text { fMRI seed-based connectivity analysis: } \\
\text { - Resting state (hyperconnectivity) } \\
\text { - right pulvinar (PV)- right postcentral, supramarginal gyrus (SMG) } \\
\text { - Pre-cuneus-right pre-central gyrus/frontal eye fields } \\
\text { - V1-SMG and post-central gyrus } \\
\text { - Resting state (hypoconnectivity): } \\
\text { - right V5-posterior cingulate cortex. } \\
\text { - cerebellar seed- PCC and medial precuneus } \\
\text { - PV- bilateral caudate nuclei } \\
\text { - Task (hyperconnectivity) } \\
\text { - right PV-right lingual gyrus } \\
\text { - right V1- right V5, postcentral,precentral gyri, SMG, premotor cortex, supplementary motor cortex(SMA), FEF } \\
\text { - V5-right cuneus, Brodmann 17, } 18 \text { and 19, the FEF, SMG, premotor cortex, SMA, superior parietal lobule (SPL) and } \\
\text { intraparietal sulcus, V1 } \\
\text { - pMCC/PCC-bilateral medial pre-cuneus, PCC. } \\
\text { - cerebellar seed-RSPL, lat pre-cuneus, post-central gyrus } \\
\text { - Task (hypoconnectivity) } \\
\text { - V5-posterior cingulate cortex, bilat medial pre-cuneus, TPJ und AG }\end{array}$ \\
\hline & $\begin{array}{l}\text { Aldusary et al. } \\
\text { (69) }\end{array}$ & $19 / 16$ & $\begin{array}{l}\text { Resting state fMRI (seed-based): } \\
\text { - Hyperconnectivity: } \\
\text { - Left anterior inferior temporal gyrus-left posterior temporal fusiform gyrus } \\
\text { - Right anterior inferior temporal gyrus-right anterior temporal fusiform gyrus } \\
\text { - Left posterior superior temporal gyrus-right inferior occipito-temporal gyrus } \\
\text { - Left angular gyrus-left lateral pre-frontal cortex } \\
\text { - Right frontal eye field-right angular gyrus } \\
\text { - Left inferior frontal gyrus-left middle frontal gyrus }\end{array}$ \\
\hline $\begin{array}{l}{\left[{ }^{123}\right] \text {-IMP }} \\
\text { SPECT }\end{array}$ & $\begin{array}{l}\text { Shibata et al. } \\
(74)\end{array}$ & $\begin{array}{l}3 \\
\text { patients/no } \\
\text { controls }\end{array}$ & $\begin{array}{l}{\left[{ }^{123} \text { I]-IMP single-photon emission computed tomography }\right.} \\
\text { 1. Right temporooccipital hypoperfusion } \\
\text { 2. Mild bilateral frontal hypoperfusion } \\
\text { 3. No pathological findings }\end{array}$ \\
\hline
\end{tabular}

(15), paresthesia (37), and tremor (15) have been described, but the latter without a further differentiation of the subtype.

Psychiatric comorbidities seem to be quite common in VSS, especially affective disorders such as anxiety, up to $50 \%$ (13), and depression, up to $58 \%$ (13). Patients also report cognitive complaints in the form of "brain fog" (16) and concentration problems (4). Another frequent symptom is derealization (16), which can be linked to the above-mentioned psychiatric disorders or appear independently (38).

\section{Secondary Forms of VS and Triggers}

Ophthalmological or radiological routine findings are without pathological findings in most cases $(4,11,16,17,39)$. There are some case reports of patients with positive visual phenomena similar to those in VS, which turned out to be caused by ocular pathology, such as birdshot retinopathia $(16,40)$, but also diseases leading to an extensive visual impairment like in a patient described by Mehta et al. who had advanced macular atrophy with cystic retinal degeneration and developed CharlesBonnet syndrome with VS in addition (16). This might indicate that sensory deafferentation could play a role in the development of VS.

Cerebral diseases, especially affecting the occipital and/or temporal brain regions, such as a pinealis cyst (with amelioration of symptoms after removal) (41), a case of Creutzfeldt-Jakob disease (42), idiopathic intracranial hypertension, posterior 
cortical atrophy, multiple sclerosis (16), or glutamic acid decarboxylase antibody syndrome (43), have been described to cause secondary VS or even VSS. Catarci reported one patient who developed permanent left-sided VS in the context of an acute occlusion of the right posterior cerebral artery (44).

In some patients, systemic infections, seizures of probable temporal or occipital origin $(16,45)$, concussions $(16,45)$, hormonal changes, drugs such as steroids (16), antidepressants (46), or isotretinoin (47) were suspected triggers for VSS.

An important differential diagnosis is "hallucinogen persisting preception disorder" (HPPD): It is a chronic syndrome characterized by a spontaneous recurrence of perceptual/visual disturbances that are similar to those generated during an intoxication with a hallucinogenic drug (DSM V) (48). In the cohort of Puledda et al. (11), there were no significant differences concerning the phenotype of the VSS between a HPPD group (70 patients) and the VSS group (1,061 patients). The HPPD patients had similar comorbidities (migraine and tinnitus) (11). Van Dongen et al. did not find a difference in VSS manifestation (intensity) in a group of 24 HPPD patients and 37 VSS patients, but the VSS patients were significantly more affected by migraine (49). Drugs that were reported to cause VSS in HPPD were ecstasy, cannabis, psilocybin mushrooms, amphetamine, 4-FMP, 3-MMC, 2C-B, ketamine, and nitrous oxide (49). This is important since these recreational drugs can obviously "trigger" a VSS-like disorder in subjects who per se do not have increased risk due to the non-increased prevalence of migraine and migraine aura.

\section{Evidence for Therapeutic Effect of Medication}

The current data indicate a possible effect of lamotrigine, which is an anti-seizure medication also shown to be preventive in migraine with visual aura (50). It inhibits voltage-gated sodium and calcium channels in the central nervous system, restricting the firing rate of cortical neurons and thereby lowering cortical excitability (50). Fekete et al. reported a case of VSS with complete remission under the therapy with lamotrigine (51), while other studies found only a small effect (in the sense of an amelioration) in a small number of patients $(13,52)$. There are single case reports of a decrease in symptom severity after the use of mydriatics (53) and a remission under amitriptyline (54). Antipsychotic drugs (55), glucocorticoids, beta-blocker, acetazolamide (17), other antidepressants (56) and antiseizure medications, benzodiazepines, migraine prophylaxis, and even ketamine were tried with a few cases of partial improvement, but mostly no benefit (16).

\section{Neurobehavioral Measures}

Since the diagnosis of the VSS is at the moment solely based on subjective measures, there have been several attempts to find a specific neurobehavioral or electrophysiological signature. Solly et al. utilized oculomotor tasks to examine 64 VSS patients and 23 controls (one subgroup of VSS patients with migraine, one without migraine, and a healthy control group).
They demonstrated significantly quicker prosaccades in VSS patients as well as more errors in incongruencies between precue and target (37). A follow-up study with 67 patients examined interfering or conflicting saccade tasks (of which one was internally cued) again showing quicker prosaccadic movements and more errors in choosing (also internally cued) antisaccadic (contralateral) marks. This suggests that the underlying problem might go beyond the management of attention given to external stimuli, but rather include a coordination problem on the level of the saccadic control system (with hyperactivity in the prosaccade system) (57).

Three groups examined visual perception thresholds in VSS patients demonstrating decreased spatial contrast sensitivity (17), reduced center-surround contrast suppression, elevated luminance increment thresholds on a textured background (58), and significantly more difficulties recognizing image orientations specifically at a flickering frequency of $15 \mathrm{~Hz}$ (59).

In another study, VS patients showed normal color and rapid flicker sensitivity but a delayed dilatation after the initial constriction of the pupil after the presentation of a chromatic stimulus (60). The latter could indicate a longer afferent stimulus persistence (60) or an autonomic dysregulation (17). A limitation of this study (60) was the small number of patients and controls included.

\section{Electrophysiological Evidence}

As an approach to study the visual pathway, several groups recorded visual evoked potentials. Eren et al. compared a group of 18 VSS patients to matched healthy controls and migraine patients. They demonstrated an increased N145 latency, which could indicate a disturbance in the secondary visual areas. There were also reduced N75-P100 amplitudes representing the afferent visual pathways between the retina and the primary visual cortex (61). Two groups investigated habituation of the P100-response after repetitive stimulation in VS patients compared to healthy controls. Yildiz et al. included a subgroup of VSS patients with migraine and a subgroup of VSS patients without migraine (24), while Luna et al. examined only one VSS patient who did not have the diagnosis of a migraine (62). In both papers, decreased habituation indicated increased cortical excitability over the afferent visual pathways, especially the primary visual cortex (V1) and possibly a disturbance of negative feedback mechanisms $(24,62)$.

In an occipital TMS application, the phosphene threshold was lower in the VSS group compared to healthy controls (24). These findings, too, might indicate neuronal hyperexcitability in the visual pathway (24). In contrast, Eren et al. could not find a significant effect of TMS application over the visual cortex on letter recognition (63). Grey et al. applied TMS at 10 and $10+$ $1 \mathrm{~Hz}$ over occipital brain areas during 20 sessions in nine patients. They found an improvement of VS intensities after the $10+1 \mathrm{~Hz}$ application, but no significant difference of the comparison to Sham or $10 \mathrm{~Hz}$ (64). 


\section{Brain Imaging}

Another attempt to find the causes underlying the VSS is looking for functional and structural correlates in neuronal imaging studies. Schankin et al. combined $\left[{ }^{18} \mathrm{~F}\right]-2$-fluoro-2deoxy-D-glucose positron emission tomography (FDG-PET) in 20 VSS patients (matched with 20 healthy controls) with MRI in 17 patients and controls (65). Puledda et al. performed MRI (magnetic resonance imaging) with seed-based MR spectroscopy in 24 patients and an equal number of matched controls (6668). Aldusary et al. compared a cohort of 19 VSS patients to 16 controls using MRI (69). In all three studies, patients and controls were matched for age and sex but not migraine (Table 1).

In the FDG-PET analysis, focal hypermetabolism was demonstrated in the secondary visual area of the right gyrus lingualis presumably as a correlate of neuronal hyperactivity in this area (65). A matching increase in gray matter volume (in MRI volumetry) was shown in the adjacent right fusiform gyrus, and in the lingual gyrus itself in the cohort of Adusary et al. (69). Interestingly, the symptom duration positively correlated with gray matter volume (GMV) in both lingual gyri (69). This might reflect differences caused by neuronal plasticity (70). Additionally, the seed-based MR spectroscopy by Puledda et al. showed a lactate peak in this area correlating negatively with the BOLD response as a sign of anaerobic (possibly inefficient or abnormal) metabolism (67).

There are hints that other areas of the visual system might be implicated as well. Puledda et al. found increased gray matter volume (whole brain voxel-wise volumetry) in the left primary (seed-based) and secondary visual cortex (V2 and V5) (66). Beyond that, the group showed in a seed-based functional MRI (fMRI) analysis of the regions of interest in the right hemisphere that there was increased connectivity between the thalamus and the lingual gyrus at tasks while the connection between thalamus and basal ganglia resting state was decreased. V1 and V5 showed hyperconnectivity between each other and with widespread cortical regions (somatosensory and motor areas including the supramarginal gyrus and frontal eye field) during stimulation. V5 had a decreased connectivity to the posterior cingulate cortex at rest, which is part of the default mode network (68). Aldusary et al. found seed-based fMRI resting state hyperconnectivity between extrastriate visual and other temporal brain regions (69).

Other non-visual brain areas seem to be affected as well. The response to a stimulation mimicking VS resulted in a reduced BOLD response compared to baseline over the bilateral anterior insulae $(67,71)$. Aldusary showed resting state hyperconnectivity between pre-frontal and parietal brain regions (69). Schankin et al. demonstrated FDG hypometabolism in the right superior temporal gyrus and the left inferior parietal lobule without associated structural alterations (65). Volumetrically, increased gray matter volume in the right middle temporal gyrus, parahippocampal gyrus, the left superior temporal gyrus and right anterior cingulate cortex (65), and cerebellum (seed-based) crus I/lobule V of the left hemisphere (66) was found.

There is one case report of a patient with VSS and another one about a patient with a prolonged migraine aura with VS demonstrating increased diffusivity in the occipital lobe, as well as the temporal lobes including the dorsal visual stream, the ventral visual stream, and the integrative visual stream $(72,73)$. Shibata et al. performed [ $\left.{ }^{123} \mathrm{I}\right]$-IMP single-photon emission computed tomography in one VSS patient showing right occipital and temporal hypoperfusion with and minimal bifrontal hypoperfusion in a second case (74).

\section{DISCUSSION}

The clinical picture of VSS consists of a characteristic constellation of visual symptoms that might be attributed to different components of the visual pathway.

In patients with migraine, the involvement of the right lingual gyrus has been shown in photophobia (75). In VSS, we find imaging, metabolic, and possibly electrophysiological evidence of increased metabolism, excitability, and connectivity in and with this area. The fMRI findings by Puledda et al. (68) indicate an implication of the visual motion area V5 with increased connectivity within and beyond the visual cortices with multiple brain areas. These give further insights into the pathophysiological mechanisms underlying the VS phenomenon and could be connected to the dynamic nature of this visual misperception (68).

The perception of entoptic phenomena indicates a "filtering" problem since these partly "physiological" but irrelevant sensations are enhanced in VSS (22).

On another level, nyctalopia and the increased luminance threshold (58) and decreased contrast sensitivity (17) might be connected by a lack of inhibitory feedback mechanisms in the visual system, normally allowing to extract relevant information and suppress visual noise.

Palinopsia might also be a phenomenon of disinhibition, leading to the repetitive perception of a dysfunctional visual memory (76). In this regard, previous case reports show that palinopsia can be caused by occipital, parietal, or temporal lesions mostly of the right hemisphere (76).

The electrophysiological evidence suggests hyperexcitability of the primary visual cortex $(24,61)$ possibly explaining the perception of photopsia.

Puledda et al. found in their fMRI analysis a disturbance in thalamostriatal connectivity while thalamocortical connections were increased (68). The thalamostriatal loop was shown to be relevant in visual learning (77), and it was demonstrated in an animal model that a disruption of this system leads to a decrease in visual precision $(68,77)$. The thalamus is linked to widespread areas of the cortex including the primary and secondary visual areas of the brain (78). This is consistent with the hypothesis that VSS could be a form of thalamocortical dysrhythmia (15).

On the other hand, the decreased BOLD response over the anterior insulae (67) as well as the increased gray matter volume over the right anterior cingulate cortex (65) could indicate a disturbance in the so-called salience network. This network plays a central role in the steering of attention, coordination between large-scale networks in task-related functions (79), and the selection of relevant stimuli (80). Furthermore, the main 
hubs of the salience network and the thalamus are closely interconnected (81).

There are some limitations of studies on VSS that have to be considered also in this review. Many of the earlier studies were done in smaller groups. For many imaging and most electrophysiological findings, patients have not been matched for migraine or migraine aura. This is relevant since a high comorbidity would confound the findings by also investigating migraine instead of VSS alone. In some of the case reports and neurobehavioral tests, the ICHD-3 criteria for VSS have not been applied. Similarly, patients might have been affected by the symptom VS, instead of VSS. Furthermore, intake of medication or previous drug use has not been reported in all studies. Future studies have to apply the ICHD 3 criteria for VSS and should include a reasonably sized number of patients that are matched for migraine and migraine aura. To study pharmacological and non-pharmacological treatment approaches, randomized and controlled trials are needed.

\section{CONCLUSION}

Already from a clinical perspective, the symptoms of VSS cannot be attributed to a single brain area or a functional unit. Rather, there is evidence of a network disorder that might manifest as a disturbance in coordination or interaction between different parts of the visual system causing a loss of inhibitory modulation and thereby hyperactivity in the primary and secondary visual cortex.

VSS seems to be a spectrum disorder with different degrees of severity, e.g., defined by the number of additional symptoms (11). There is an association with tinnitus thought to be caused by cortical hyperexcitability and production of a phantom sensation (82). Several patients may suffer from comorbid fibromyalgia, the classical centralized pain disorder with hypersensitivity to external stimuli (83). There are potentially common underlying mechanisms (doi: 10.1111/head.14213).

Migraine is the most prevalent comorbidity in patients affected by VSS. Migraine can be seen as a sensory gating

\section{REFERENCES}

1. Liu GT, Schatz NJ, Galetta SL, Volpe NJ, Skobieranda F, Kosmorsky GS. Persistent positive visual phenomena in migraine. Neurology. (1995) 45:664-8. doi: 10.1212/WNL.45.4.664

2. Jäger H, Giffin N, Goadsby P. Diffusion-and perfusion-weighted MR imaging in persistent migrainous visual disturbances. Cephalalgia. (2005) 25:323-32. doi: 10.1111/j.1468-2982.2004.00858.x

3. Simpson JC, Goadsby PJ, Prabhakar P. Positive persistent visual symptoms (visual snow) presenting as a migraine variant in a 12-year-old girl. Pediatr Neurol. (2013) 49:361-3. doi: 10.1016/j.pediatrneurol.2013. 07.005

4. Schankin CJ, Maniyar FH, Digre KB, Goadsby PJ. 'Visual snow' - a disorder distinct from persistent migraine aura. Brain. (2014) 137:1419-28. doi: 10.1093/brain/awu050

5. Bartleson J. Transient and persistent neurological manifestations of migraine. Stroke. (1984) 15:383-6. doi: 10.1161/01.STR.15.2.383

6. Viana M, Tronvik EA, Do TP, Zecca C, Hougaard A. Clinical features of visual migraine aura: a systematic review. J Headache Pain. (2019) 20:64. doi: 10.1186/s10194-019-1008-x disorder with a persisting hypersensitivity to internal and external stimuli even in the interictal state $(84,85)$. It is associated with more severe manifestations of VSS. It can be hypothesized that migraine could cause a pre-disposition to develop a persistent sensory network disorder like VSS or, based on case reports, migraine attacks could even be triggers.

Other triggers include medications, seizure, trauma, and recreational drug use, when HPPD is counted as a form of secondary VSS. Secondary VSS caused by structural lesions seem to involve different posterior areas of the brain. One potential mechanism of secondary and triggered forms might be an imbalance of this network.

Understanding the communication within this network and how its modulation might lead to VSS is crucial if treatment strategies should be developed for this currently almost untreatable condition.

\section{DATA AVAILABILITY STATEMENT}

The original contributions presented in the study are included in the article, further inquiries can be directed to the corresponding author.

\section{AUTHOR CONTRIBUTIONS}

AK: conceived and designed the research, collected the data, organized the papers, and wrote the paper. CJS: conceived and designed the research, organized the papers, and wrote the paper and checked the manuscript. All authors contributed to the article and approved the submitted version.

\section{FUNDING}

This research was funded by the Visual snow Initiative: funding of the publication fee. CJS was supported by Baasch Medicus Foundation.

7. Schankin CJ, Maniyar FH, Sprenger T, Chou DE, Eller M, Goadsby PJ. The relation between migraine, typical migraine aura and "visual snow". Headache. (2014) 54:957-66. doi: 10.1111/head.12378

8. Sastre-Ibáñez M, Santos-Bueso E, Porta-Etessam J, García-Feijoo J. Visual snow: report of three cases. J Fr Ophtalmol. (2015) 38:e157-8. doi: 10.1016/j.jfo.2014.06.030

9. Olesen J. Headache Classification Committee of the International Headache Society (IHS) the international classification of headache disorders, asbtracts. Cephalalgia. (2018) 38:1-211. doi: 10.1177/0333102417738 202

10. Page MJ, Moher D, Bossuyt PM, Boutron I, Hoffmann TC, Mulrow CD, et al. PRISMA 2020 explanation and elaboration: updated guidance and exemplars for reporting systematic reviews. BMJ. (2021) 372:osf.io/gwdhk. doi: 10.31222/osf.io/gwdhk

11. Puledda F, Schankin C, Goadsby PJ. Visual snow syndrome: a clinical and phenotypical description of 1,100 cases. Neurology. (2020) 94:e564-74. doi: 10.1212/WNL.0000000000008909

12. Viana M, Puledda F, Goadsby PJ. Visual snow syndrome: a comparison between an Italian and British population. Eur J Neurol. (2020) 27:2099-101. doi: 10.1111/ene.14369 
13. Van Dongen RM, Waaijer LC, Onderwater GL, Ferrari MD, Terwindt GM. Treatment effects and comorbid diseases in 58 patients with visual snow. Neurology. (2019) 93:e398-403. doi: 10.1212/WNL.0000000000007825

14. Bessero A-C, Plant GT. Should "visual snow" and persistence of after-images be recognised as a new visual syndrome? J Neurol Neurosurg Psychiatry. (2014) 85:1057. doi: 10.1136/jnnp-2013-306827

15. Lauschke JL, Plant GT, Fraser CL. Visual snow: a thalamocortical dysrhythmia of the visual pathway? J Clin Neurosci. (2016) 28:123-7. doi: 10.1016/j.jocn.2015.12.001

16. Mehta DG, Garza I, Robertson CE. Two hundred and forty-eight cases of visual snow: a review of potential inciting events and contributing comorbidities. Cephalalgia. 2021:0333102421996355. doi: 10.1177/0333102421996355

17. Yoo Y-J, Yang HK, Choi J-Y, Kim J-S, Hwang J-M. Neuro-ophthalmologic findings in visual snow syndrome. J Clin Neurol. (2020) 16:646. doi: $10.3988 /$ jen.2020.16.4.646

18. Critchley M. Types of visual perseveration: "paliopsia" and "illusory visual spread”. Brain. (1951) 74:267-99. doi: 10.1093/brain/74.3.267

19. Abert B, Ilsen PF. Palinopsia. Optometry. (2010) 81:394-404. doi: 10.1016/j.optm.2009.12.010

20. Abraham HD. Visual phenomenology of the LSD flashback. Arch Gen Psychiatry. (1983) 40:884-9. doi: 10.1001/archpsyc.1983.01790070074009

21. Friedman B. Observations on entoptic phenomena. Arch Ophthalmol. (1942) 28:285-312. doi: 10.1001/archopht.1942.00880080103012

22. Cipolletta S, Beccarello A, Galan A. A psychological perspective of eye floaters. Qual Health Res. (2012) 22:1547-58. doi: 10.1177/1049732312456604

23. Sommerville DN. Vitrectomy for vitreous floaters: analysis of the benefits and risks. Curr Opin Ophthalmol. (2015) 26:173-6. doi: 10.1097/ICU.0000000000000150

24. Yildiz FG, Turkyilmaz U, Unal-Cevik I. The clinical characteristics and neurophysiological assessments of the occipital cortex in visual snow syndrome with or without migraine. Headache. (2019) 59:484-94. doi: $10.1111 /$ head.13494

25. Riva CE, Petrig B. Blue field entoptic phenomenon and blood velocity in the retinal capillaries. J Opt Soc Am. (1980) 70:1234-8. doi: 10.1364/JOSA.70.001234

26. Bowen SF. Retinal entoptic phenomena: some diagnostic use. Arch Ophthalmol. (1963) 69:551-5. doi: 10.1001/archopht.1963.00960040557003

27. Eren OE, Ruscheweyh R, Straube A, Schankin CJ. Quantification of photophobia in visual snow syndrome: a case-control study. Cephalalgia. (2020) 40:393-8. doi: 10.1177/0333102419896780

28. Virdee J, Mollan SP. Photopsia. Prac Neurol. (2020) 20:415-9. doi: 10.1136/practneurol-2019-002460

29. Mehra D, Le PH. Physiology, Night Vision (2019).

30. Digre KB, Brennan KC. Shedding light on photophobia. $J$ Neuroophthalmol. Tampa: StatPearls Publishing (2012) 32:68-81. doi: 10.1097/WNO.0b013e3182474548

31. Perenboom MJ, Najafabadi AHZ, Zielman R, Carpay JA, Ferrari MD. Quantifying visual allodynia across migraine subtypes: the Leiden Visual Sensitivity Scale. Pain. (2018) 159:2375. doi: 10.1097/j.pain.0000000000001343

32. Graber M, Goadsby P, Scutelnic A, Schankin C. Natural course of Visual Snow Syndrome: a long-term follow-up study. Eur J Neurol. (2020) 27:162 (EPR1103). doi: 10.1111/ene.14307

33. Hodak J, Fischer U, Bassetti CL, Schankin CJ. Episodic visual snow associated with migraine attacks. JAMA Neurol. (2020) 77:392-3. doi: 10.1001/jamaneurol.2019.4050

34. Schankin CJ, Puledda F, Goadsby PJ. Visual snow syndrome: is it normal or a disorder - and what to do with patients? Eur J Neurol. (2020) 27:2393-5. doi: 10.1111 /ene.14436

35. Kondziella D, Olsen MH, Dreier JP. Prevalence of visual snow syndrome in the UK. Eur J Neurol. (2020) 27:764-72. doi: 10.1111/ene.14150

36. Beyer U, Gaul C. Visual snow. Der Nervenarzt. (2015) 86:1561-5. doi: 10.1007/s00115-015-4401-y

37. Solly EJ, Clough M, McKendrick AM, Foletta P, White OB, Fielding J. Ocular motor measures of visual processing changes in visual snow syndrome. Neurology. (2020) 95:e1784-91. doi: 10.1212/WNL.0000000000010372
38. Hunter EC, Phillips ML, Chalder T, Sierra M, David AS. Depersonalisation disorder: a cognitive-behavioural conceptualisation. Behav Res Ther. (2003) 41:1451-67. doi: 10.1016/S0005-7967(03)00066-4

39. Tegetmeyer H. [Visual snow syndrome: symptoms and ophthalmological findings]. Klinische Monatsblatter fur Augenheilkunde. (2017) 234:713-8. doi: 10.1055/s-0042-109701

40. Patel RC, Vitale AT, Creel DJ, Digre KB. Not all that flickers is snow. J Neuro Ophthalmol. (2021) 41:e97-9. doi: 10.1097/WNO.0000000000000935

41. Weiß S, Rohde V, Hautmann X, Schittkowski M. Visual-snowsyndrom - Wenn die Augen rauschen. Der Ophthalmologe. (2021) 118. doi: 10.1007/s00347-021-01398-1. [Epub ahead of print].

42. Chen BS, Lance S, Lallu B, Anderson NE. Visual snow: not so benign. J Clin Neurosci. (2019) 64:37-9. doi: 10.1016/j.jocn.2019.03.023

43. Piquet AL, Khan M, Warner JEA, Wicklund MP, Bennett JL, Leehey MA, et al. Novel clinical features of glycine receptor antibody syndrome. A series of 17 cases. Neurol Neuroimmunol Neuroinflamm. (2019) 6:e592. doi: 10.1212/NXI.0000000000000592

44. Catarci T. Occipital ischaemic stroke after visual snow phenomenon-a case report. Cephalalgia. (2021) 41:871-4. doi: 10.1177/0333102420985444

45. Polster T, Schaefer S, Panzer A. Visual snow-two adolescents with a new syndrome different from migraine or epilepsy. Neuropediatrics. (2019) 50:S155. doi: 10.1055/s-0039-1698175

46. Eren OE, Schöberl F, Schankin CJ, Straube A. Visual snow syndrome after start of citalopram-novel insights into underlying pathophysiology. Eur J Clin Pharmacol. (2021) 77:271-2. doi: 10.1007/s00228-020-02996-9

47. Berkowitz E, River Y, Digre K, Tiosano B, Kesler A. Visual snow: a case series from Israel. Case Rep Ophthalmol. (2020) 11:205-11. doi: 10.1159/000508602

48. Orsolini L, Valchera A, Papanti D, Vecchiotti R, De Berardis D. The "Endless Trip": psychopathology and psychopharmacology in the Hallucinogen Persisting Perception Disorder (HPPD). Eur Psychiatry. (2020) 33:S310-S. doi: 10.1016/j.eurpsy.2016.01.1060

49. van Dongen RM, Alderliefste GJ, Onderwater GLJ, Ferrari MD, Terwindt GM. Migraine prevalence in visual snow with prior illicit drug use (Hallucinogen Persisting Perception Disorder) versus without. Eur J Neurol. (2021) 28:26318. doi: 10.1111/ene.14914

50. Buch D, Chabriat H. Lamotrigine in the prevention of migraine with aura: a narrative review. Headache. (2019) 59:1187-97. doi: 10.1111/head.13615

51. Fekete R. Visual snow syndrome successfully treated with lamotrigine: case report. (P4.129). Neurology. (2018) 90(15 Suppl.):129.

52. Unal-Cevik I, Yildiz FG. visual snow in migraine with aura: further characterization by brain imaging, electrophysiology, and treatment case report. Headache: The J Head Face Pain. (2015) 55:1436-41. doi: $10.1111 /$ head. 12628

53. Coleman W, Sengupta S, Boisvert CJ. A case of visual snow treated with phenylephrine. Headache. (2021) 61:792-3. doi: 10.1111/head.14118

54. Wentzell S, Ryan M. Visual snow syndrome: a case report and new treatment option. Clin Med Rev Case Rep. (2018) 5:246. doi: 10.23937/2378-3656/1410246

55. Prashar KS, Titus AM, Kinkhabwala V, Hirsch AR. 148 visual snow defeats guardians of the galaxy volume 2: unremitting pixelation despite three-dimentional stereoscopic film. CNS Spectrums. (2018) 23:91-2. doi: 10.1017/S1092852918000421

56. Eren O, Schankin CJ. Mirtazapine for treatment of visual snow syndrome: a case series with insights into pathophysiology and therapy. Clin Transl Neurosci. (2020) 4:2514183X20925695. doi: 10.1177/2514183X20925 695

57. Solly EJ, Clough M, McKendrick AM, Foletta P, White OB, Fielding J. Eye movement characteristics provide an objective measure of visual processing changes in patients with visual snow syndrome. Sci Rep. (2021) 11:1-10. doi: 10.1038/s41598-021-88788-2

58. McKendrick AM, Chan YM, Tien M, Millist L, Clough M, Mack $\mathrm{H}$, et al. Behavioral measures of cortical hyperexcitability assessed in people who experience visual snow. Neurology. (2017) 88:1243-9. doi: 10.1212/WNL.0000000000003784

59. Eren O, Eggert T, Ruscheweyh R, Straube A, Schankin C. Visual contrast threshold at $15 \mathrm{~Hz}$ is able to confirm visual snow syndrome in individual patients (S20. 005). AAN Enterprises (2019). 
60. Alissa R, Bi W, Bessero A, Plant G, Barbur J. Vision in subjects with hyperawareness of afterimages and "visual snow". Acta Ophthalmol. (2012) 90:F066. doi: 10.1111/j.1755-3768.2012.F066.x

61. Eren O, Rauschel V, Ruscheweyh R, Straube A, Schankin CJ. Evidence of dysfunction in the visual association cortex in visual snow syndrome. Ann Neurol. (2018) 84:946-9. doi: 10.1002/ana.25372

62. Luna S, Lai D, Harris A. Antagonistic relationship between VEP potentiation and gamma power in visual snow syndrome. Headache. (2018) 58:138-44. doi: 10.1111/head.13231

63. Eren OE, Ruscheweyh R, Rauschel V, Eggert T, Schankin CJ, Straube A. Magnetic suppression of perceptual accuracy is not reduced in visual snow syndrome. Front Neurol. (2021) 12:498. doi: 10.3389/fneur.2021.658857

64. Grey V, Klobusiakova P, Minks E. Can repetitive transcranial magnetic stimulation of the visual cortex ameliorate the state of patients with visual snow? Bratislavske Lekarske Listy. (2020) 121:395-9. doi: 10.4149/BLL_2020_064

65. Schankin CJ, Maniyar FH, Chou DE, Eller M, Sprenger T, Goadsby PJ. Structural and functional footprint of visual snow syndrome. Brain. (2020) 143:1106-13. doi: 10.1093/brain/awaa053

66. Puledda F, Bruchhage M, O’Daly O, Ffytche D, Williams SC, Goadsby PJ. Occipital cortex and cerebellum gray matter changes in visual snow syndrome. Neurology. (2020) 95:e1792-9. doi: 10.1212/WNL.0000000000010530

67. Puledda F, Ffytche D, Lythgoe DJ, O'Daly O, Schankin C, Williams SC, et al. Insular and occipital changes in visual snow syndrome: a BOLD fMRI and MRS study. Ann Clin Transl Neurol. (2020) 7:296-306. doi: $10.1002 / \operatorname{acn} 3.50986$

68. Puledda F, O'Daly O, Schankin C, Ffytche D, Williams SC, Goadsby PJ. Disrupted connectivity within visual, attentional and salience networks in the visual snow syndrome. Hum Brain Mapp. (2021) 42:2032-44. doi: $10.1002 / \mathrm{hbm} .25343$

69. Aldusary N, Traber GL, Freund P, Fierz FC, Weber KP, Baeshen A, et al. Abnormal connectivity and brain structure in patients with visual snow. Front Hum Neuroscience. (2020) 14:476. doi: 10.3389/fnhum.2020.582031

70. Kutch JJ, Ichesco E, Hampson JP, Labus JS, Farmer MA, Martucci KT, et al. Brain signature and functional impact of centralized pain: a multidisciplinary approach to the study of chronic pelvic pain (MAPP) network study. Pain. (2017) 158:1979-91. doi: 10.1097/j.pain.0000000000001001

71. Puledda F, Lythgoe D, O’Daly O, Schankin C, Williams S, Goadsby P. Functional and metabolic changes in visual snow syndrome: a combined BOLD fMRI and MRspectroscopy study. Neurology. (2020) 7(3):296-306.

72. Belvís R, Ramos R, Villa C, Segura C, Pagonabarraga J, Ormazabal $\mathrm{I}$, et al. Brain apparent water diffusion coefficient magnetic resonance image during a prolonged visual aura. Headache. (2010) 50:1045-9. doi: $10.1111 /$ j.1526-4610.2010.01672.x

73. Latini F, Fahlström M, Marklund N, Feresiadou A. White matter abnormalities in a patient with visual snow syndrome: New evidence from a diffusion tensor imaging study. Eur J Neurol. (2021) 28:2789-93. doi: 10.1111 /ene.14903

74. Shibata M, Tsutsumi K, Iwabuchi Y, Kameyama M, Takizawa T, Nakahara T, et al. [123I]-IMP single-photon emission computed tomography imaging in visual snow syndrome: a case series. Cephalalgia. (2020) 40:1671-5. doi: 10.1177/0333102420950454

75. Denuelle M, Boulloche N, Payoux P, Fabre N, Trotter Y, Géraud G. A PET study of photophobia during spontaneous migraine attacks. Neurology. (2011) 76:213-8. doi: 10.1212/WNL.0b013e3182074a57
76. Gersztenkorn D, Lee AG. Palinopsia revamped: a systematic review of the literature. Surv Ophthalmol. (2015) 60:1-35. doi: 10.1016/j.survophthal.2014.06.003

77. Kato S, Kuramochi M, Kobayashi K, Fukabori R, Okada K, Uchigashima $\mathrm{M}$, et al. Selective neural pathway targeting reveals key roles of thalamostriatal projection in the control of visual discrimination. J Neurosci. (2011) 31:17169-79. doi: 10.1523/JNEUROSCI.4005-1 1.2011

78. López-Bendito G. Development of the thalamocortical interactions: past, present and future. Neuroscience. (2018) 385:67-74. doi: 10.1016/j.neuroscience.2018.06.020

79. Chiong W, Wilson SM, D'Esposito M, Kayser AS, Grossman SN, Poorzand $\mathrm{P}$, et al. The salience network causally influences default mode network activity during moral reasoning. Brain. (2013) 136:1929-41. doi: 10.1093/brain/awt066

80. Uddin LQ. Salience processing and insular cortical function and dysfunction. Nat Rev Neurosci. (2015) 16:55-61. doi: 10.1038/nrn3857

81. Peters SK, Dunlop K, Downar J. Cortico-striatal-thalamic loop circuits of the salience network: a central pathway in psychiatric disease and treatment. Front Syst Neurosci. (2016) 10:104. doi: 10.3389/fnsys.2016.00104

82. De Ridder D, Elgoyhen AB, Romo R, Langguth B. Phantom percepts: tinnitus and pain as persisting aversive memory networks. Proc Natl Acad Sci USA. (2011) 108:8075-80. doi: 10.1073/pnas.1018466108

83. Siracusa R, Paola RD, Cuzzocrea S, Impellizzeri D. Fibromyalgia: pathogenesis, mechanisms, diagnosis and treatment options update. Int J Mol Sci. (2021) 22:3891. doi: 10.3390/ijms22083891

84. Goadsby PJ, Holland PR, Martins-Oliveira M, Hoffmann J, Schankin C, Akerman S. Pathophysiology of migraine: a disorder of sensory processing. Physiol Rev. (2017) 97:553-622. doi: 10.1152/physrev.00034.2015

85. Peng K-P, May A. Migraine understood as a sensory threshold disease. Pain. (2019) 160:1494-501. doi: 10.1097/j.pain.0000000000001531

Conflict of Interest: CJS received scientific support, travel support and/or honoraria from Novartis, Eli Lilly, TEVA Pharmaceuticals, Lundbeck, Allergan, Almirall, Amgen, MindMed, Grünenthal. He received research grants from German Migraine and Headache Society, Eye on Vision Foundation, and Baasch Medicus Foundation. CJS is part-time employee at Zynnon.

The remaining author declares that the research was conducted in the absence of any commercial or financial relationships that could be construed as a potential conflict of interest.

Publisher's Note: All claims expressed in this article are solely those of the authors and do not necessarily represent those of their affiliated organizations, or those of the publisher, the editors and the reviewers. Any product that may be evaluated in this article, or claim that may be made by its manufacturer, is not guaranteed or endorsed by the publisher.

Copyright (c) 2021 Klein and Schankin. This is an open-access article distributed under the terms of the Creative Commons Attribution License (CC BY). The use, distribution or reproduction in other forums is permitted, provided the original author(s) and the copyright owner(s) are credited and that the original publication in this journal is cited, in accordance with accepted academic practice. No use, distribution or reproduction is permitted which does not comply with these terms. 